\title{
Comparação entre Critérios de Seleção de Precocidade Sexual e a Associação destes com Características de Crescimento em Bovinos Nelore ${ }^{1}$
}

\author{
Carlos Dario Ortiz Peña², Sandra Aidar de Queiroz ${ }^{3}$, Luiz Alberto Fries ${ }^{4}$
}

\begin{abstract}
RESUMO - Neste trabalho estimaram-se parâmetros genéticos do perímetro escrotal (PE), PE corrigido para idade (PEi) e PEi corrigido para peso corporal (PEip) de bovinos Nelore, comparando-se a classificação dos animais pelas DEPs, segundo o critério adotado. Além disso, estimaram-se a correlação genética existente entre as três expressões do PE e o ganho médio diário pré-desmama, o ganho médio diário pós-desmama, o número de dias para ganhar 160 kg após o nascimento e o número de dias para ganhar 240 kg após a desmama. As informações foram obtidas nos Registros de Produção da Associação Paraguaia dos Criadores de Nelore, entre 1986 e 1997, em 110 rebanhos. As estimativas dos componentes de variância e herdabilidade foram obtidas pelo Método da Máxima Verossimilhança Restrita, usando modelos animais uni característica. As herdabilidades estimadas foram 0,41; 0,40; e 0,47 para PE, PEi e PEip, respectivamente, todas indicando possibilidade de progresso genético por seleção. A estimativa de correlação genética entre PEi e PEip $(0,62)$ foi moderada e os valores obtidos para as correlações entre as características de precocidade sexual e de crescimento foram de pequena magnitude. As estimativas de correlações de rank das DEPs foram 0,98 para PE e PEi e 0,59 para PEi e PEip. Os resultados evidenciaram a ocorrência de mudanças na classificação dos melhores animais, quando se considerou ou não o peso vivo na correção do PE. A utilização do perímetro escrotal corrigido para idade e peso corporal como critério de seleção evitaria ou reduziria a resposta correlacionada com tamanho adulto superior, trazendo maiores progressos genéticos na precocidade sexual.
\end{abstract}

Palavras-chave: correlação genética, correlação de rank, dias para ganhar 160 kg, ganho médio diário, herdabilidade, perímetro escrotal corrigido para idade e peso

\section{Comparison of Selection Criteria for Sexual Precocity and their Association with Growth Traits in Nellore Cattle}

\begin{abstract}
In this study genetic parameters of scrotal circumference (SC), SC corrected for age $\left(\mathrm{SC}_{/ \mathrm{a}}\right)$ and $\mathrm{SC}_{/ \mathrm{a}}$ corrected for live weight $\left(\mathrm{SC}_{/ \mathrm{aw}}\right)$ of Nellore Cattle were estimated and comparisons of the rank based on EPDs of the above criteria were made. In addition, the genetic correlations between SC measurements and growth (preweaning average daily gain, number of days to gain $160 \mathrm{~kg}$ after birth, post weaning average daily gain and number of days to gain $240 \mathrm{~kg}$ after weaning) were estimated. The information came from the Paraguayan Nellore Breeders Association Production Registry, obtained from 1986 to 1997, in 110 herds. Restricted Maximum Likelihood using one-trait animal models were used to obtain the variance components and heritability estimates. The heritability estimates were $0.41,0.40$, and 0.47 for SC, $\mathrm{SC}_{/ \mathrm{a}}$ and $\mathrm{SC}_{/ \mathrm{aw}}, \mathrm{respectively}_{\mathrm{f}}$ all of them indicating the possibility of genetic improvement by selection. The estimate of genetic correlation between $\mathrm{SC}_{/ \mathrm{a}}$ and $\mathrm{SC}_{/ \mathrm{aw}}(0.62) \mathrm{was}$ moderate and the obtained values for the correlations between the sexual precocity and growth traits were of small magnitude. The rank correlations between EPDs estimates were 0.98 for $\mathrm{SC}_{\text {and }} \mathrm{SC}_{/ \mathrm{a}}$ and $0.59 \mathrm{for} \mathrm{SC}_{/ \mathrm{a}}$ and $\mathrm{SC}_{/ \mathrm{aw}}$. The results showed changes in the classification of animals selected when live weight was or was not considered in the SC correction. The selection criterion scrotal circumference corrected for age and live weight $\left(\mathrm{SC}_{/ \mathrm{aw}}\right)$ could reduce a correlated response with larger adult size, while obtaining substantial improvement in sexual precocity.
\end{abstract}

Key Words: average daily gain, days to gain $160 \mathrm{~kg}$ from birth to weaning, genetic correlation, heritability, rank correlation, scrotal circumference corrected to age and live weight

\section{Introdução}

O perímetro escrotal (PE) é, em animais jovens, indicador útil do tamanho testicular, da capacidade de produção espermática, das características físicas do sêmen, da idade à puberdade e, também, da fertilidade dos machos e das fêmeas aparentadas (meio-irmãs e filhas) (COULTER e KELLER, 1982; TOELLE e ROBINSON, 1985; ALENCAR et al., 1993; SILVA et al., 1993; ARIAS e SLOBODZIAN, 1998; e GRESSLER et al., 1998). Em razão de o perímetro escrotal ser facilmente mensurável e possuir alta repetibilidade, tem merecido grande atenção como critério de seleção de touros, visando ao melhoramento

\footnotetext{
${ }^{1}$ Parte da dissertação de mestrado do primeiro autor para obtenção do título de Mestre em Zootecnia na FCAV - UNESP.

2 Engenheiro-agrônomo autônomo. Bolsista CAPES convênio PEC - PG - Brasil-Paraguai. E.mail: carlos.ortiz@cad.com.py

${ }^{3}$ Departamento de Zootecnia - FCAV - UNESP - Jaboticabal. Bolsista do CNPq. E.mail: saquei@fcav.unesp.br

4 Gensys Consultores Associados Ltda. Professor visistante - CAPES - DZ/FCAV - UNESP. E.mail: fries@fcav.unesp.br
} 
dos rebanhos no aspecto reprodutivo.

UNANIAM (1997) constatou o aparecimento dos primeiros espermatozóides vivos a partir dos 10,6 meses de idade, variando até os 16 meses, sendo a média de 13,6 meses com 22,9 cm de PE para bovinos Nelore. Segundo VIEIRA et al. (1988) e SILVA et al. (1993), o crescimento mais intenso do testículo ocorre nas idades mais próximas à puberdade. Portanto, a tomada da medida do PE neste período é estratégica para avanços genéticos em fertilidade e precocidade sexual (LÔBO et al., 1994; DALFARRA et al., 1998).

Em touros jovens Nelore e Polled Hereford, DAL-FARRA et al. (1998) e BRITO (1997), respectivamente, constataram efeitos significativos linear e quadrático da idade e do peso corporal do animal sobre o PE. Assim, os autores recomendaram a correção do PE para os efeitos de idade e peso do animal, a fim de obter-se progresso genético em precocidade sexual, evitando-se o aumento do tamanho adulto do animal.

LÔBO et al. (1994) e ELER et al. (1996), trabalhando com 11.070 e 31.114 informações de PE de touros jovens Nelore, estimaram em 0,45 e 0,52 a herdabilidade dessa característica, respectivamente. Os valores estimados pelos autores foram altos, confirmando a existência de variabilidade genética aditiva, o que permite obter-se progresso genético na seleção de touros jovens que seriam potencialmente bons quanto à produção espermática futura.

Além da precocidade sexual, assume importância conhecer a inter-relação desta com a precocidade de crescimento. Assim, outros parâmetros a considerar são as correlações fenotípicas e genéticas entre as características reprodutivas e as de desenvolvimento ponderal. MARTINS FILHO et al. (1994) e ELER et al.(1996) estimaram correlação genética baixa $(0,18)$ entre o PE e o peso à desmama (PD) de bovinos Nelore. ALENCAR e VIEIRA (1989), LÔBO et al. (1994) e QUIRINO e BERGMANN (1997) reportaram valores de moderados a altos para a correlação genética $(0,51 ; 0,44$; e 0,64 , respectivamente) entre PE e peso ao sobreano, sugerindo a possibilidade de selecionar, simultaneamente, estas características nos programas de melhoramento.

Os objetivos desse trabalho foram estimar parâmetros genéticos para o perímetro escrotal mensurado ao sobreano; comparar a característica perímetro escrotal ajustando-se ou não para idade e peso corporal; e verificar a relação existente entre as características de precocidade sexual e de crescimento.

\section{Material e Métodos}

Nesta pesquisa foram estudadas 7458 informações do perímetro escrotal (PE), mensurado ao sobreano, de bovinos da raça Nelore criados no Paraguai. Além da medida observada, o PE foi, também, corrigido para a idade do animal (PEi) e para a idade e o peso do animal (PEip). O PE foi corrigido para 570 dias de idade e $310 \mathrm{~kg}$ de peso vivo, baseando-se nas médias de idade e peso dos animais ao sobreano. A correção do PE para idade e peso ao sobreano exigiu a padronização do peso ao sobreano para 570 dias de idade (P570). Foram estimados fatores de correção multiplicativos específicos para o PE para intervalos de 530 a 610 dias de idade e para 160 a $590 \mathrm{~kg}$ de P570, de acordo com ORTIZ PEÑA et al. (2000).

Também foram utilizadas 39.409 informações de ganho médio diário pré-desmama (GMD) e número de dias para ganhar $160 \mathrm{~kg}$ após o nascimento (D160), e 16.393 observações de ganho médio diário pósdesmama (GMP) e número de dias para ganhar $240 \mathrm{~kg}$ após a desmama (D240).

O GMD foi pré-ajustado para a idade do animal na desmama (205 dias), data juliana de nascimento e idade da vaca ao parto, de acordo com os fatores de correção utilizados pela ABCZ (GENSYS, 1996). D160 foi obtido a partir do GMD pré-ajustado para os fatores mencionados acima (D160 $=160 /$ GMD).

O grupo de contemporâneos (GC) foi composto por animais nascidos no mesmo rebanho, ano, estação, do mesmo sexo, pesados e medidos no mesmo dia na desmama e no sobreano, e pertencentes ao mesmo grupo de manejo da desmama e do sobreano.

Para a verificação da conexão entre os GC, utilizou-se o programa MILC (Matriz de Incidência, Laços Genéticos e Conectabilidade) elaborado por FRIES e ROSO (1997).

As estimativas dos componentes de variância e herdabilidade foram obtidas pelo Método da Máxima Verossimilhança Restrita e os valores genéticos (VG), por meio de modelos animais uni característica, utilizando-se o programa computacional Multi Traits Derivative Free Restricted Maximum Likelihood (MTDFREML), segundo BOLDMAN et al. (1993), com equações de modelos mistos específicas para cada característica.

O modelo completo adotado na fase de desmama foi: $\mathrm{Y}=\mathrm{X} \beta+\mathrm{Za}+\mathrm{Mm}+\mathrm{Wp}+\mathrm{e}$

em que: $\mathrm{Y}$ é vetor das observações pré-ajustadas (GMD ou D160); X, matriz de incidência associada aos efeitos fixos; $\beta$, vetor dos efeitos fixos (GC da 
desmama); Z, M, W, matrizes de incidência associadas aos efeitos aleatórios; a, vetor do efeito genético aditivo direto do animal (aleatório); m, vetor do efeito genético aditivo materno (aleatório); p, vetor do efeito de ambiente permanente (aleatório); e, vetor dos resíduos aleatórios, não observáveis.

O modelo utilizado para as características pósdesmame foi semelhante ao anterior, sendo o vetor $Y$ correspondente às observações de GMP, D240, PE, PEi e PEip, mas, para estas análises, não foram incluídos os efeitos aleatórios materno e de ambiente permanente.

Nos modelos considerados para as avaliações genéticas, tem-se que: $\mathrm{E}(\mathrm{Y})=\mathrm{Xb}, \mathrm{E}(\mathrm{a})=0, \mathrm{E}(\mathrm{m})=0$, $\mathrm{E}(\mathrm{p})=0, \mathrm{E}(\mathrm{e})=0$; com $\operatorname{Var}(\mathrm{a})=\mathrm{A} \sigma^{2}{ }_{\mathrm{A}}, \operatorname{Var}(\mathrm{m})=$ $\mathrm{A} \sigma^{2}{ }_{\mathrm{M}}, \operatorname{Var}(\mathrm{p})=\mathrm{INV} \sigma_{\text {ap }}^{2}$ e $\operatorname{Var}(\mathrm{e})=\mathrm{IN} \sigma^{2}{ }_{\mathrm{e}}$, sendo que A é o numerador da matriz de parentesco; $\sigma^{2}{ }_{\mathrm{A}}$, a variância genética aditiva direta; $\sigma^{2}{ }_{\mathrm{M}}$, a variância genética aditiva materna; $\sigma^{2}$ ap a variância de ambiente permanente fornecido pela mãe; $\sigma^{2}$, a variância residual, $\mathrm{NV}$, número de vacas; $\mathrm{N}$, número de observações; e I, uma matriz identidade.

As correlações entre os VG das características estudadas foram estimadas pelo procedimento CORR (Pearson) (SAS, 1996). Segundo VAN VLECK et al. (1987), a estimativa de correlação entre valores genéticos é a própria definição de correlação genética. As diferenças esperadas nas progênies (DEP) dos animais foram calculadas dividindo-se os VG por dois. As DEPs para D160 e D240 foram multiplicadas por menos um (-1), de maneira que pudessem representar dias a "mais" de precocidade (ALBUQUERQUE e FRIES, 1998). Pelo procedimento CORR (Spearman) (SAS, 1996), foram estimadas as correlações entre os ranks das DEP para os animais avaliados.

Com o objetivo de analisar o impacto dos critérios de seleção PEi e PEip na escolha de reprodutores, os animais foram classificados nas categorias touros $(\mathrm{T})$, vacas em produção $(\mathrm{V})$ e produtos da última safra (P). Os critérios para classificação dos indivíduos em cada uma dessas categorias foram: a) categoria $\mathrm{T}$ - reprodutores com mais de cinco filhos avaliados; b) categoria $\mathrm{V}$ - vacas com última parição registrada em 1994; e c) categoria P - animais nascidos a partir de 1995.

A comparação entre os critérios de seleção PEi e PEip foi feita utilizando-se a classificação das DEPs dos indivíduos, em ordem decrescente. Foram comparados os dez melhores touros nos dois critérios e observadas as coincidências no posicionamento dos animais dentro de classes percentuais de $10 \mathrm{em} 10 \%$, denominadas decas. Foi avaliada a coincidência de posição entre os 10\% melhores indivíduos (Deca 1), para todas as três categorias e os dois critérios de seleção e, visando, respectivamente, à seleção e ao descarte, as coincidências de posição entre os $20 \%$ melhores (Decas 1 e 2) e 20\% piores (Decas 9 e 10) para as categorias V e P. As quantidades ou percentagens de não-coincidência devem ser interpretadas como o número ou a proporção de animais que não seriam selecionados por um critério de seleção, quando utilizado o outro critério e vice-versa.

A coincidência de decas dos animais, de acordo com as características PEi e PEip, também foi investigada. As mudanças de classificação dos animais entre decas foram avaliadas por meio da diferença entre decas. Os valores obtidos foram agrupados em classes que variaram de zero, quando não houve diferença entre decas, a sete, quando esta foi máxima. Assim, na classe zero, pode-se encontrar tanto coincidência de ordenamentos individuais quanto diferenças de, no máximo, o valor correspondente a 9,9\% da população analisada. Na classe 1 , os ordenamentos podem diferir de, no mínimo, uma posição individual e, no máximo, posições correspondentes à quantidade de $19,9 \%$ da população avaliada. Para as classes maiores que 1 , as diferenças em posições individuais podem ser de, no mínimo, a quantidade da população correspondente à diferença de deca em questão menos 1 e, no máximo, a quantidade da população correspondente à diferença de deca em questão mais 1 (pe.: dif. deca $=4 ; 4-1=3$; dif. min. de posição individual $=29,9 \%$ da pop.; $4+1=5$; dif. max. de classificação indiv. $=49,9 \%$ da pop.).

\section{Resultados e Discussão}

Na Tabela 1 encontram-se as estatísticas descritivas para o perímetro escrotal, idade e peso dos animais ao sobreano. Os valores apresentados estão dentro dos limites descritos na literatura para esta raça. Pode-se observar que, embora as amplitudes tenham aumentado gradualmente, os desvios-padrão e coeficientes de variação do PE diminuíram com o ajuste para a idade do animal, reduzindo-se em maior grau, quando passou a ajustar, também, para o peso corporal. Além disso, observa-se que a variação é bem maior para a característica P570 do que para a idade do animal aos 570 dias.

As estimativas dos componentes de variância e coeficientes de herdabilidade obtidas para o períme- 
Tabela 1 - Estatísticas descritivas das características perímetro escrotal, peso e idade ao sobreano de bovinos da raça Nelore Table 1 - Descritive statistics for the traits scrotal circumference, yearling weight and yearling age of Nellore cattle

\begin{tabular}{|c|c|c|c|c|c|c|}
\hline $\begin{array}{l}\text { Característica } \\
\text { Trait }\end{array}$ & $\mathrm{N}$ & $\begin{array}{l}\text { Média } \\
\text { Mean }\end{array}$ & $\mathrm{DP}( \pm)$ & $\mathrm{CV}(\%)$ & $\begin{array}{l}\text { Mínimo } \\
\text { Minimum }\end{array}$ & $\begin{array}{l}\text { Máximo } \\
\text { Maximum }\end{array}$ \\
\hline Idade (dias) & 7458 & 574 & 19 & 3,28 & 519 & 663 \\
\hline \multicolumn{7}{|l|}{ Age (days) } \\
\hline P570 (kg) & 7458 & 310 & 57 & 18,30 & 154 & 594 \\
\hline $\mathrm{PE}(\mathrm{cm})$ & 7458 & 25,71 & 3,54 & 13,76 & 13 & 40 \\
\hline $\operatorname{PEi}(\mathrm{cm})$ & 7458 & 25,55 & 3,49 & 13,68 & 13,24 & 41,18 \\
\hline PEip $(\mathrm{cm})$ & 7458 & 25,92 & 3,04 & 11,73 & 13,33 & 41,76 \\
\hline
\end{tabular}

$\overline{\mathrm{DP}}=$ desvio-padrão; $\mathrm{CV}$ = coeficiente de variação; P570 = peso padronizado aos 570 dias de idade; PE = perímetro escrotal observado; $\mathrm{PEi}$ = perímetro escrotal corrigido para 570 dias de idade; e PEip = perímetro escrotal corrigido para 570 dias de idade e 310 kg aos 570 dias de idade.

$D P=$ standard deviation; $C V=$ coefficient of variation; $P 570=$ body weight corrected to 570 days old $; P E=$ observed scrotal circumference; $P E i=$ scrotal circumference corrected to 570 days old and PEip = scrotal circumference corrected to 570 days old and $310 \mathrm{~kg}$.

tro escrotal podem ser observadas na Tabela 2. Verifica-se que os coeficientes de herdabilidade estimados para todas as expressões do PE foram altos, indicando a possibilidade de melhorar estas características por meio de seleção. As estimativas obtidas concordam com as relatadas na literatura, sendo menores que as estimadas nesta raça e idade, por ELER et al. (1996) e QUIRINO e BERGMANN (1997), e superiores às obtidas por GRESSLER et al. (1998). Constata-se que a herdabilidade do PE ajustado para idade e peso (PEip) foi maior $(0,47)$ que as demais estimativas ( $\mathrm{PE}=0,41$ e $\mathrm{PEi}=0,40$ ) e verifica-se, também, a ocorrência de mudanças nas proporções dos componentes de variância, havendo maior redu-

Tabela 2 - Componentes de variância e herdabilidade $\left(\mathrm{h}^{2}\right)$ estimados para o perímetro escrotal (PE), em bovinos da raça Nelore

Table 2 - Estimated variance components and heritability $\left(h^{2}\right)$ of scrotal circumference (PE) of Nellore cattle

\begin{tabular}{lccc}
\hline $\begin{array}{l}\text { Estimativa } \\
\text { Estimate }\end{array}$ & \multicolumn{3}{c}{$\begin{array}{c}\text { Característica } \\
\text { Trait }\end{array}$} \\
\cline { 2 - 4 } & $\mathrm{PE}$ & $\mathrm{PEi}(\mathrm{a})$ & $\mathrm{PEip}(\mathrm{b})$ \\
\hline$\sigma_{A}^{2}$ & 2,9349 & 2,7854 & 2,8449 \\
$\sigma_{e}^{2}$ & 4,2810 & 4,1057 & 3,1751 \\
$\sigma_{P}^{2}$ & 7,2159 & 6,8911 & 6,0201 \\
$\mathrm{~h}^{2}$ & 0,4100 & 0,4000 & 0,4700 \\
\hline
\end{tabular}

(a) corrigido para 570 dias de idade; (b)corrigido para 570 dias de idade e $310 \mathrm{~kg}$ aos 570 dias de idade; $\sigma_{A}^{2}=$ variância genética aditiva; $\sigma_{e}^{2}=$ variância de ambiente; $\sigma_{P}^{2}=$ variância fenotípica; $\mathrm{h}^{2}=$ herdabilidade.

(a) corrected to 570 days old; (b)corrected to 570 days old and $310 \mathrm{~kg}$; $\sigma_{A}^{2}=$ genetic additive variance; $\sigma_{e}^{2}=$ environmental variance; $\sigma_{P}^{2}=$ phenotypic variance; $h^{2}=$ heritability. ção na variância ambiental, quando considerados a idade e o peso do animal, que foi a principal responsável pelas diferenças nas estimativas de herdabilidade. A redução na variância de ambiente mostra a importância da correção do PE para estas duas grandes fontes de variação não genéticas, permitindo a melhor identificação dos animais sexualmente mais precoces, conforme BRITO (1997) e DAL-FARRA (1998). Pode-se argumentar, ainda, que a correção para o peso removeu outros efeitos ambientais que agiram sobre o desenvolvimento do animal, inclusive o testicular, mas não foram adequada ou suficientemente controlados ou modelados.

$\mathrm{Na}$ Tabela 3 encontram-se as estimativas das correlações genéticas entre as três expressões do PE e as características de crescimento pré e pós-desmama. As correlações genéticas estimadas entre PE e PEi e as de desenvolvimento ponderal foram de moderadas a baixas $(|0,28|$ a $|0,33|)$, apresentando magnitudes inferiores com as características de pré-desmama (GMD e D160) e, em ambas as fases, pré-desmama e sobreano, maiores magnitudes com as expressões de ganho médio diário (GMD e GMP). Já as estimativas das correlações genéticas entre PEip e as expressões do crescimento foram baixas e com sinais invertidos tanto na pré-desmama como no sobreano. Estes resultados vão de encontro aos relatados por ALENCAR e VIEIRA (1989), LÔBO et al. (1994) e QUIRINO e BERGMANN (1997). As estimativas obtidas neste trabalho sugerem que os genes envolvidos na determinação da velocidade de crescimento, na pré e pós-desmama, estariam pouco relacionados com os que expressam a precocidade sexual das três maneiras mensuradas nesta pesquisa. Além disso, os animais com maiores DEPs para PEip não necessa- 
Rev. bras. zootec.

Tabela 3 - Estimativas de correlação genética entre características de precocidade sexual e precocidade de crescimento, em bovinos da raça Nelore

Table 3 - Genetic correlation estimates of sexual and growth precocity traits of Nellore cattle

\begin{tabular}{lllll}
\hline $\begin{array}{l}\text { Precocidade sexual } \\
\text { Sexual precocity }\end{array}$ & \multicolumn{5}{c}{$\begin{array}{c}\text { Precocidade de crescimento } \\
\text { Growth precocity }\end{array}$} \\
\cline { 2 - 5 } & GMD & D160 & GMP & D240 \\
\hline $\mathrm{PE}^{(\mathrm{a})}$ & $0,30^{*}$ & $-0,28^{*}$ & $0,33^{*}$ & $-0,27^{*}$ \\
$\mathrm{PEi}^{(\mathrm{b})}$ & $0,30^{*}$ & $-0,29^{*}$ & $0,32^{*}$ & $-0,27^{*}$ \\
$\mathrm{PEip}^{(\mathrm{c})}$ & $-0,16^{*}$ & $0,14^{*}$ & $-0,28^{*}$ & $0,22^{*}$ \\
\hline
\end{tabular}

(a) perímetro escrotal; (b) PE corrigido para 570 dias de idade; (c) PE corrigido para 570 dias de idade e $310 \mathrm{~kg}$ aos 570 dias de idade; GMD = ganho médio diário pré-desmama; D160 = número de dias para ganhar $160 \mathrm{~kg}$ após o nascimento; GMP = ganho médio diário pós-desmama; D240 = número de dias para ganhar $240 \mathrm{~kg}$ após a desmama. ${ }^{*} \mathrm{P}<0,0001$.

(a) scrotal circumference; (b) corrected to 570 days old; (c) corrected to 570 days old and $310 \mathrm{~kg} ; G M D=$ pre-weaning average daily gain; $D 160=$ number of days to gain $160 \mathrm{~kg}$ after birth; GMP = post weaning average daily gain; D240 = number of days to gain $240 \mathrm{~kg}$ after weaning .

riamente seriam os de maiores taxas de crescimento. Menor grau de associação genética entre as características de precocidade sexual e de crescimento poderia permitir, dependendo do objetivo de seleção, maior ênfase seletiva na precocidade sexual, sem elevar, em demasia, a taxa de crescimento e o tamanho adulto das vacas de cria. Cabe ressaltar, entretanto, que parte desta associação negativa entre as características pode ter sido construída pelo fato de o peso fazer parte do denominador do PEip. Assim, outros estudos envolvendo esta e outras características indicativas da conformação do peso corporal, em machos Nelore, seriam importantes para elucidar estas associações entre genes de precocidade sexual e de crescimento.

Constam da Tabela 4 os resultados obtidos para as correlações genéticas e de classificação (rank) das DEPs dos animais para as diferentes expressões do PE. Diferenças muito pequenas foram constatadas entre as correlações de rank dos animais classificados para as DEPs de PE e PEi. Verifica-se que a correlação dos ranks para PE e PEi foi quase igual a um, caindo para 0,58 a estimada entre os de PE e PEip e para 0,59 a obtida entre os ranks para PEi e PEip, sugerindo grande modificação na posição de classificação dos animais, quando se opta por corrigir o PE para idade e peso. Pode-se verificar, também, que as correlações genéticas estimadas entre PE, PEi e PEip foram todas altas, sendo a estimativa da correlação entre o PE e PEi quase igual a 1,0, ocorrendo queda acentuada para aquela entre PEip e $\operatorname{PE}(0,61)$ ou PEi $(0,62)$. Estes resultados indicam que as três expressões desta característica estariam governadas em grande medida, pelos mesmos genes em iguais direção e sentido, porém com magnitudes médias $(0,61$ a 0,62$)$, quando se considera o peso corporal do animal no ajuste do PE.

Na Figura 1 é apresentada a dispersão das DEPs (acima) e dos "ranks" (abaixo) dos touros avaliados para PEi e PEip. Observa-se grande dispersão das DEPs, com leve concentração de animais com DEPs médias. O grau destas alterações pode ser conferido na dispersão dos ranks dos touros, onde percebe-se que houve grandes mudanças ao longo de todas as posições dos touros classificados para PEi e PEip. Verifica-se, ainda, concentração um pouco mais acentuada dos animais posicionados nos extremos, sugerindo relação com tendência positiva entre ambas as características, embora esta não seja bem clara, devido à grande dispersão das DEPs.

Listando-se os 10 primeiros touros classificados para PEi e PEip, tem-se o apresentado na Tabela 5, que, também, evidencia a ocorrência de alterações nas posições para as DEPs destes animais, segundo o critério adotado. Constata-se que, entre os 10 touros que seriam selecionados para PEi, cinco deles também o seriam pelo critério PEip. Entretanto, os animais classificados em $4^{\mathrm{a}}$ e $8^{\mathrm{a}}$ posições para PEi ("AW" e "BA") ocupariam entre $186^{\mathrm{a}}$ e $108^{\mathrm{a}}$ para PEip, respectivamente, e o $2^{\circ}$ touro para PEip ("BD") passaria a ser o $206^{\circ}$ posicionado para PEi.

Tabela 4 - Estimativas de correlação genética (acima da diagonal), de classificação (rank) dos animais avaliados (abaixo da diagonal) e médias ( \pm d.p.) das DEPs (diagonal principal) para o perímetro escrotal (PE) de bovinos Nelore

Table 4 - Genetic correlation estimates between the three measures of Scrotal circumference (PE) (above diagonal), EPD ranking correlation of the three measures of Scrotal circumference (bellow diagonal) and Scrotal circumference EPD means (on diagonal) of Nellore cattle

\begin{tabular}{llll}
\hline$D E P S$ & \multicolumn{1}{c}{ PE } & \multicolumn{1}{c}{ PEi $^{(a)}$} & \multicolumn{1}{c}{ PEip $^{(\mathrm{b})}$} \\
PE & $0,04( \pm 0,47)$ & $0,98^{*}$ & $0,61 *$ \\
PEi & $0,98^{*}$ & $0,03( \pm 0,47)$ & $0,62^{*}$ \\
PEip & $0,58^{*}$ & $0,59^{*}$ & $0,002( \pm 0,49)$ \\
\hline
\end{tabular}

(a) corrigido para 570 dias de idade; ${ }^{(b)}$ corrigido para 570 dias de idade e $310 \mathrm{~kg}$ de peso aos 570 dias de idade; $\mathrm{N}=13.536$; ${ }^{*} \mathrm{P}<0,0001$

a) corrected to 570 days old; (b) corrected to 570 days old and $310 \mathrm{~kg}$. 

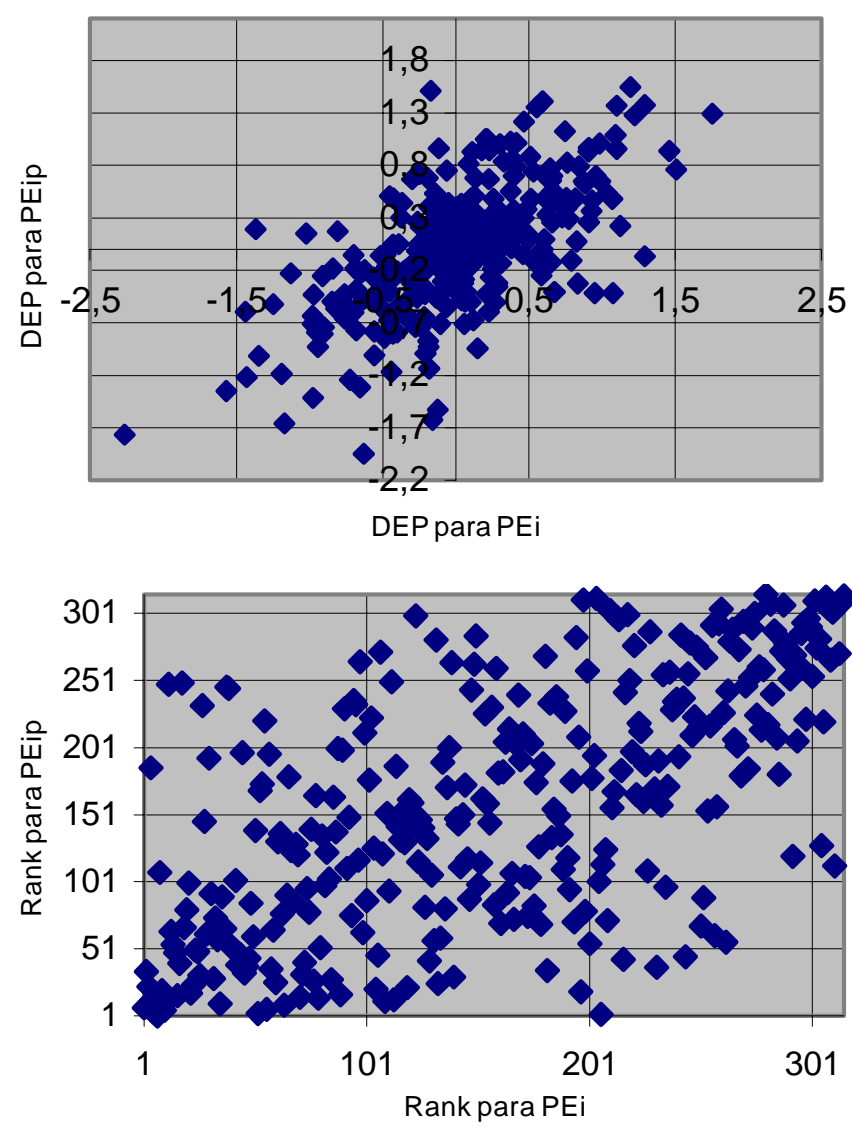

Figura 1 - Dispersão das DEPs e dos ranks para os touros Nelore avaliados para PE corrigido para 570 dias de idade (PEi) e para 570 dias de idade e $310 \mathrm{~kg}$ (PEip).

Figure 1 - Dispersion of Nelore sires EPDs and ranks for scrotal circumference corrected to 570 days old (PEi) and scrotal circumference corrected to 570 days old and $310 \mathrm{~kg}$ (PEip).
Na Figura 2 apresenta-se a freqüência de diferenças entre decas verificada para as classificações dos touros avaliados para os critérios PEi e PEip. Pode-se observar que a maior parte destes touros (33\%) apresentou uma deca de diferença entre a classificação para PEi e PEip, englobando diferenças que variaram de uma posição individual dentro da deca até posições referentes a $19,9 \%$ do total de animais avaliados. Também constatou-se que seis touros $(2 \%)$ apresentaram sete decas de diferença. $\mathrm{O}$ fato de que, na prática, a escolha do animal possa ser feita segundo sua classificação pela deca faz com que a utilização de um ou outro critério de seleção para PE influencie a intensidade de uso desse reprodutor e, também, o seu valor econômico.

$\mathrm{Na}$ Tabela 6 apresentam-se os graus de nãocoincidência entre as classificações do PEi e PEip dos animais, por categoria. Sua análise evidencia que, tanto para os extratos de escolha dos reprodutores (10 e $20 \%$ melhores) quanto para o de descarte de animais (20\% piores), aproximadamente, $50 \%$ ou mais dos animais não foram coincidentes segundo os critérios de correção do PE. Os resultados de não-coincidência indicam que $50 \%$ ou mais dos animais que seriam utilizados por um critério de seleção não o seriam pelo outro, confirmando o grande impacto que poderia ter a correção prévia para estas fontes de variação, na procura de precocidade sexual por meio da seleção baseada no PE.

Os resultados obtidos neste trabalho indicam que a seleção por um ou outro critério traria consequiências diferentes a longo prazo nos programas de melhoramento. A utilização do PE corrigido para

Tabela 5 - Classificação dos 10 primeiros touros Nelore, de acordo com a DEP para o PE corrigido para idade (PEi) e para idade e peso (PEip)

Table 5 - Ranking of top ten Nellore bulls, according to Scrotal circumference corrected to 570 days old (PEi) and scrotal circumference corrected to 570 days old and $310 \mathrm{~kg}$ (PEip) EPDs

\begin{tabular}{|c|c|c|c|c|}
\hline \multirow[b]{2}{*}{$\begin{array}{l}\text { Posição } \\
\text { Rank }\end{array}$} & \multicolumn{2}{|c|}{ PEi } & \multicolumn{2}{|c|}{ PEip } \\
\hline & $\begin{array}{l}\text { Identidade animal } \\
\text { Animal identification }\end{array}$ & $\begin{array}{l}\text { Posição PEip } \\
\text { Rank PEip }\end{array}$ & $\begin{array}{l}\text { Identidade animal } \\
\text { Animal identification }\end{array}$ & $\begin{array}{l}\text { Posição PEi } \\
\text { Rank PEi }\end{array}$ \\
\hline$\overline{1 \underline{0}}$ & AT & 7 & $\mathrm{AZ}$ & 7 \\
\hline $2^{\mathrm{O}}$ & $\mathrm{AU}$ & 34 & $\mathrm{BD}$ & 206 \\
\hline $3^{\underline{o}}$ & $\mathrm{AV}$ & 23 & $\mathrm{BE}$ & 52 \\
\hline 40 & AW & 186 & $\mathrm{AX}$ & 5 \\
\hline $5 \underline{0}$ & $\mathrm{AX}$ & 4 & $\mathrm{BC}$ & 10 \\
\hline 60 & AY & 8 & $\mathrm{BF}$ & 56 \\
\hline $7 \stackrel{0}{0}$ & AZ & 1 & AT & 1 \\
\hline $8^{\circ}$ & BA & 108 & $\mathrm{AY}$ & 6 \\
\hline $9 \underline{0}$ & $\mathrm{BB}$ & 20 & BG & 64 \\
\hline $10^{\mathrm{o}}$ & $\mathrm{BC}$ & 5 & BH & 35 \\
\hline
\end{tabular}


Rev. bras. zootec.

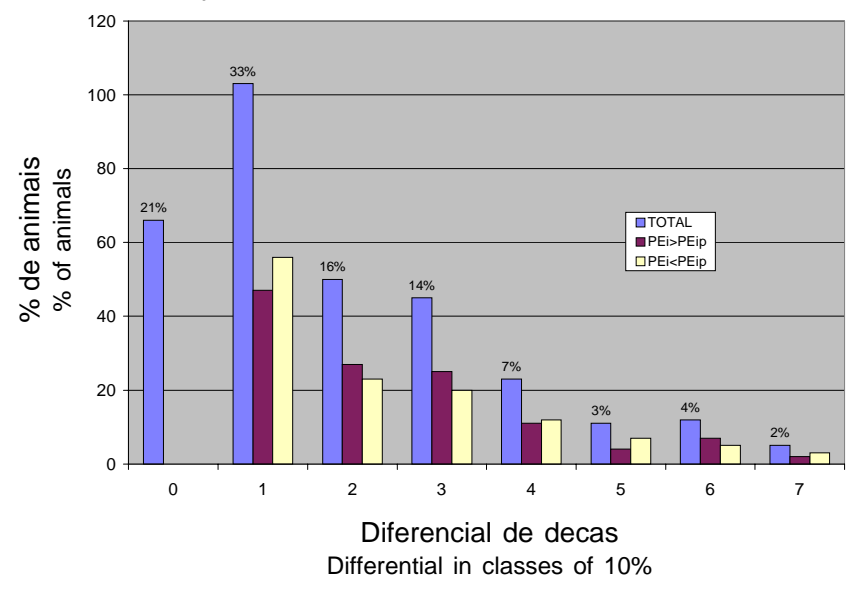

Figura 2 - Freqüência da diferença entre decas para perímetro escrotal corrigido para 570 dias de idade (PEi) e perímetro escrotal corrigido para 570 dias de idade e $310 \mathrm{~kg}$ (PEip), dos touros Nelore.

Figure 2 - Differences in ranking position (in classes of 10\%) of Nelore sires EPDs for scrotal circumference corrected to 570 days old (PEi) and scrotal circumference corrected to 570 days old and $310 \mathrm{~kg}$ (PEip).

Tabela 6 - Classificação não-coincidente dos animais avaliados pelo perímetro escrotal corrigido para idade (PEi) e perímetro escrotal corrigido para idade e $310 \mathrm{~kg}$ de peso (PEip), por categoria de bovinos Nelore

Table 6 - No coincident classification of Nellore bulls genetic evaluated using scrotal circumference corrected to 570 days old (PEi) and scrotal circumference corrected to 570 days old and $310 \mathrm{~kg}$ (PEip)

\begin{tabular}{|c|c|c|c|}
\hline Categoria & $\begin{array}{l}10 \% \\
\text { melhor }\end{array}$ & $\begin{array}{l}20 \% \\
\text { melhor }\end{array}$ & $\begin{array}{l}20 \% \\
\text { pior }\end{array}$ \\
\hline Class & Best $10 \%$ & Best $20 \%$ & worse $20 \%$ \\
\hline
\end{tabular}

Touros $(\mathrm{N}=315)$

Sires

\begin{tabular}{lccc}
\hline No. Total & 31 & - & - \\
$\begin{array}{l}\text { Total number } \\
\text { Não coincidentes }\end{array}$ & 19 & - & - \\
$\begin{array}{l}\text { Non coincident } \\
\% \text { (não coincidentes) }\end{array}$ & 61,29 & - & - \\
$\%$ (non coincident) & & &
\end{tabular}

Vacas mães (N=987)

Dams

\begin{tabular}{lccc}
\hline $\begin{array}{l}\text { No. Total } \\
\begin{array}{l}\text { Total number } \\
\text { Não coincidentes }\end{array}\end{array}$ & 98 & 198 & 198 \\
$\begin{array}{l}\text { Non coincident } \\
\%(\text { não coincidentes) } \\
\%(\text { non coincident) }\end{array}$ & 61,22 & 50,76 & 48,48 \\
\hline $\begin{array}{l}\text { Touros jovens (N=998) } \\
\text { Young sires }\end{array}$ & & & \\
\hline $\begin{array}{l}\text { Número total } \\
\text { Total number } \\
\text { Não coincidentes }\end{array}$ & 69 & 200 & 200 \\
$\begin{array}{l}\text { Non coincident } \\
\%(\text { não-coincidentes) } \\
\%(\text { non coincident) }\end{array}$ & 66,66 & 116 & 104 \\
\hline
\end{tabular}

idade e peso do animal como critério de seleção para precocidade sexual, quando mensurado em idades mais jovens, melhoraria o rebanho em precocidade sexual, sem, necessariamente, elevar os pesos/tamanhos adultos dos animais.

\section{Conclusões}

Os critérios de seleção perímetro escrotal observado (PE) e perímetro escrotal corrigido para idade do animal (PEi) foram coincidentes na avaliação da precocidade sexual.

A utilização dos critérios perímetro escrotal corrigido para idade e peso do animal (PEip) ou perímetro escrotal corrigido para idade do animal (PEi) poderá acarretar na escolha de indivíduos diferentes.

Espera-se maior progresso genético na precocidade sexual baseando-se a seleção no critério perímetro escrotal corrigido para idade e peso do animal (PEip).

A seleção do perímetro escrotal corrigido para idade e peso do animal (PEip), quando comparada à praticada pelo perímetro escrotal corrigido apenas para idade (PEi) ou pelo perímetro escrotal observado (PE), traria menor resposta correlacionada para velocidade de crescimento.

Os coeficientes de correlações estimados indicam a possibilidade de selecionar-se, conjuntamente, as características de precocidade sexual e de crescimento.

\section{Referências Bibliográficas}

ALBUQUERQUE, L.G., FRIES, L. A. Precocidad: estrategias de selección. In: SIMPOSIO LATINOAMERICANO PRODUCTIVIDAD EN GANADO DE CORTE, 1, 1998, Santa Cruz de la Sierra. Anais... São Paulo: DBO Sul, 1998. p.19-24.

ALENCAR, M.M., BARBOSA, P.F., BARBOSA, R.T. et al. 1993. Parâmetros genéticos para peso e circunferência escrotal em touros da raça Canchim. R. Soc. Bras. Zootec., 22(4):572-583.

ALENCAR, M.M., VIEIRA, R.C., 1989. Crescimento testicular de touros da raça Canchim. Pesq. Agropec. Bras. 24(11):1329-1333.

ARIAS, A., SLOBODZIAN, A. Análisis de los datos de los registros de la raza Nelore de Paraguay. In: CONGRESO INTERNACIONAL DE PRODUCCIÓN DE CARNE, 1, 1998, Mariano R. Alonso. Proceedings... Asunción: APCN/ BURSAL/DBO Sul, 1998. p. 51-94.

BOLDMAN, K.G., KRIESE, L.A., VAN VLECK, L.D. et al. 1993. A Manual for Use o MTDFREML. USDA-ARS. Clay Center, NE. 120p.

BRITO, F.V. Influência da idade e peso corporal sobre o perímetro escrotal em touros Hereford - estimativas de fatores de correção. In: REUNIÃO ANUAL DA SOCIEDADE BRASILEIRA DE ZOOTECNIA, 34, 1997, Juiz de Fora, Anais.. 1997. p.130-132. 
COULTER, G.H., KELLER, D.G. 1982. Scrotal circumference of young beef bulls; relationship to paired testes weight effect to breed, and predictability. Can. J. Anim. Sci., 62:133-139.

DAL-FARRA, R.A., LOBATO, J.F., FRIES, L.A. 1998. Fatores de correção do perímetro escrotal para efeitos de idade e peso ao sobreano de tourinhos Nelore. R. Bras. Zootec., 27(6):1092-96.

ELER, J. P., FERRAZ, J. B., SILVA, P. R. Estimação simultânea de parâmetros genéticos para características de importância econômica na raça Nelore, com a utilização de modelos animais. In: REUNIÃO ANUAL DA SOCIEDADE BRASILEIRA DE ZOOTECNIA, 33, 1996, Fortaleza. Anais... Viçosa: SBZ, 1996. v.1, p.99-101.

FRIES, L.A., ROSO V. M. Conectabilidade em avaliações genéticas de gado de corte: uma proposta heurística. In: REUNIÃO ANUAL DA SOCIEDADE BRASILEIRA DE ZOOTECNIA, 34, 1997, Juiz de Fora. Anais... Viçosa : SBZ, 1997. v3., p.159-161.

GENSYS Consultores e Associados. CONSIST.FOR (programa fonte que realiza consistência e ajustes para os dados da ABCZ). 1996.

GRESSLER, S.L., BERGMANN, J.A., PENNA, V.M. et al. Estudo das associações genéticas entre perímetro escrotal e características reprodutivas de fêmeas da raça Nelore. In: REUNIÃO ANUAL DA SOCIEDADE BRASILEIRA DE ZOOTECNIA, 35, 1998, Botucatu. Anais... Viçosa: SBZ, 1998. v.3, p.368-370.

LÔBO, R.B., DE LOS REYES, A., MAGNABOSCO, C. et al. Variância e covariância genética para circunferência escrotal e pesos em rebanhos Nelore. In: REUNIÃO ANUAL DA SOCIEDADE BRASILEIRA DE ZOOTECNIA, 31, 1994, Maringá, Anais... Viçosa: SBZ, 1994. p.163.

MARTINS FILHO, R., LÔBO, R.B., DE LOS REYES A. et al. Estimativas de parâmetros genéticos para a circunferência escrotal em bovinos da raça Nelore. In: REUNIÃO ANUAL DA SOCIEDADE BRASILEIRA DE ZOOTECNIA, 31, 1994, Maringá. Anais... Viçosa: SBZ, 1994. p.162.
ORTIZ PEÑA, C.D., QUEIROZ, S.A., FRIES, L.A. 2000. Estimação de fatores de correção do perímetro escrotal para idade e peso corporal de touros jovens da raça Nelore. Rev. bras. zootec., 29(6):1667-1675.

QUIRINO, C.R., BERGMANN, J.A. Herdabilidade de perímetro escrotal ajustado e não ajustado para peso corporal usando modelo animal uni e bivariado. In: REUNIÃO ANUAL DA SOCIEDADE BRASILEIRA DE ZOOTECNIA, 34, 1997, Juiz de Fora. Anais... Viçosa: SBZ, 1997. p.127-129.

SAS Institute Inc., SAS/STAT. User's Guide, version 6.11, 4.ed, v.2, Cary: SAS Institute Inc., 1996. 842p.

SILVA, A.E., DODE, M.A., UNANIAN, M.M. 1993. Avaliação da capacidade reprodutiva do touro. In: Capacidade reprodutiva do touro de corte: funções, anormalidades $e$ fatores que a influenciam. Campo Grande: EMBRAPACNPGC. p.128-11.

TOELLE, V.D., ROBISON, O.W. 1985. Estimates of genetic correlation between measurements and female reproductive traits in cattle. J. Anim. Sci., 60:89-100.

UNANIAM, M. M. A procura de marcadores de precocidade em gado Nelore. In: O NELORE DO SÉCULO XXI. Nelore Precoce: Seleção, Produção e Comercialização. SIMPÓSIO, 4, 1997, Uberaba. Anais...Uberaba: ABCZ-ABCN, 1997. p.51-57.

VAN VLECK, L.D., POLLACK, E.J., OLTENACU, E.A. 1987. Genetics for the animal breeding. New York: Freeman and Company. 391p.

VIEIRA, R.C., ALENCAR, M.M., ESTEVES, S.N. 1988. Efeito da suplementação alimentar sobre o comportamento reprodutivo de tourinhos Canchim: I. Características puberais. Pesq. Agropec. Bras., 23(1):97-102.

Recebido em: 29/05/00

Aceito em: 28/09/00 